\title{
Use of $\lambda \mathrm{pMu}$ Bacteriophages to Isolate $\lambda$ Specialized Transducing Bacteriophages Carrying Genes for Bacterial Chemotaxis
}

\author{
HISATO KONDOH, ${ }^{\prime} \dagger$ BARBARA R. PAUL, ${ }^{2} \ddagger$ AND MARTHA M. HOWE ${ }^{2 *}$ \\ Departments of Biochemistry and Genetics, ${ }^{1}$ and Department of Bacteriology, ${ }^{2}$ College of Agricultural and \\ Life Sciences, University of Wisconsin-Madison, Madison, Wisconsin 53706
}

\begin{abstract}
A general method for constructing lambda specialized transducing phages is described. The method, which is potentially applicable to any gene of Escherichia coli, is based on using Mu DNA homology to direct the integration of a $\lambda \mathrm{pMu}$ phage near the genes whose transduction is desired. With this method we isolated a lambda transducing phage carrying all 10 genes in the che gene cluster (map location, 41.5 to $42.5 \mathrm{~min}$ ). The products of the $c h e A$ and $\operatorname{tar}$ genes were identified by using transducing phages with amber mutations in these genes. It was established that tar codes for methyl-accepting chemotaxis protein II (molecular weight, 62,000) and that cheA codes for two polypeptides (molecular weights, 76,000 and 66,000 ). Possible origins of the two cheA polypeptides are discussed.
\end{abstract}

Specialized transducing phages are powerful tools in the study of gene structure and expression. They provide sources of DNA for determination of genetic maps, construction of restriction maps, determination of DNA sequences, identification of protein gene products, and detection and isolation of specific RNA's. The phage used most often for specialized transductions is phage lambda. Lambda specialized transducing phages can be isolated in vivo by aberrant excision of a $\lambda$ prophage (40) or in vitro by cloning specific DNA restriction fragments into $\lambda$ cloning vehicles (5). Isolating such phages in vivo requires that the $\lambda$ prophage be located close to the gene to be transduced. A number of methods have been developed to achieve this proximity. They include the integration of $\lambda$ prophages at secondary attachment sites (39), the integration of $\mathrm{F}^{\prime}$ factors close to the prophage attachment site (13), and the directed integration of $\lambda$ by homologous recombination between drug resistance elements present in the phage and the chromosome (19). In this work we used a method of general applicability which takes advantage of the fact that bacteriophage $\mathrm{Mu}$ integrates at random sites throughout the bacterial chromosome $(7,9,17)$. Although the $\mathrm{Mu}$ prophage itself does not form specialized transducing particles (16), it can serve as a region of homology between a $\lambda$ pMu transducing phage (8) (a $\lambda$ phage carrying a segment of Mu DNA $[6,31])$ and a $\mathrm{Mu}$ prophage inserted near the

$\dagger$ Present address: Department of Biophysics, Faculty of Science, Kyoto University, Kyoto 606, Japan.

$\ddagger$ Present address: Department of Pathology, Stanford University School of Medicine, Stanford, CA 94305. host genes of interest; consequently, the $\lambda \mathrm{pMu}$ becomes integrated near these genes. Induction of such $\lambda \mathrm{pMu}$ lysogens results in the generation of $\lambda$ specialized transducing phages carrying nearby host genes. Using this technique, we isolated a transducing phage carrying a cluster of che genes involved in chemotaxis.

The chemotactic response of Escherichia coli to a variety of attractants and repellents (33) involves a number of genes that code for proteins which can be broadly divided into three functional groups. The first group contains the receptor or binding proteins that specifically interact with attractant or repellent molecules; the second group is composed of proteins which transmit or signal the state of binding of the receptor proteins to the third group of proteins, which determine the response of the flagella to the stimulus. Within the third group of proteins are those comprising the flagellum structure itself and those which control flagellar movement. This control appears to be regulated by the degree of methylation of a group of methylaccepting chemotaxis proteins (MCPs).

In the 41.5- to 43-min region of the $E$. coli chromosome there are a large number of che and fla genes involved in this chemotactic response. Because of similar phenotypes of different mutants and the potential for complex interactions of mutations by polarity or by interactions of different proteins through direct contact and through regulatory interactions, the elucidation of the number of genes and the precise function of each gene are difficult to accomplish when only complementation and mapping analysis of mutants defective in chemotaxis are used. 
A recent approach which has been quite successful is the use of $\lambda$ transducing phages and ColE1 plasmids containing cloned segments of che region DNA to identify the proteins coded by that region and to correlate the presence of specific proteins with the ability of the phage or plasmid to complement specific host defects in chemotaxis $(41,43,44)$. In the above-mentioned work several $\lambda$ transducing phages carrying portions of the che gene cluster were isolated, and a variety of deleted derivatives were made. An analysis of the polypeptides synthesized by the deleted transducing phages suggested that the che $A$ gene directs the syntheses of two polypeptides with different molecular weights $(29,44)$ and that the tar gene codes for one of the MCPs $(23,41,44-46)$.

In this paper we report the isolation of a $\lambda$ transducing phage which carries all known genes of the che cluster and the use of amber mutant derivatives of that phage to identify che region proteins. Our results confirm the previous identifications of the cheA and tar gene products (29, $41,44,46)$.

\section{MATERIALS AND METHODS}

Bacterial and bacteriophage strains. The bacterial strains used are listed in Table 1. Strain HK1100, $\mathrm{a} \mathrm{rec}^{+}$derivative of strain MS1540 (MS1500 flaG1540; a gift from M. Simon to J. Adler), was made by mating strain MS1540 with Hfr PK25 for 45 min as described previously (22) and selecting a $\mathrm{his}^{+} \mathrm{rec}^{+}$StrA flaG:: $\mathrm{Mu}$ recombinant. Mu-resistant derivatives of strains M590 and M524 were isolated by their resistance to infection by Mu vir3057gov3261momA.

Bacteriophages $\lambda \mathrm{pMu} 526$ and $\lambda \mathrm{pMu} 624$ are $\lambda$ transducing phages which carry a small segment of $\mathrm{Mu}$
DNA originating from the immunity $(c)$ end and the $\beta$ end, respectively, of a Mu prophage $(28,31)$. They carry $\lambda$ mutations $c$ I857S7 and a small portion of the lac operon $(6,28,31)$. They are deleted for the $\lambda$ attachment site and $\lambda$ int gene and are not able to integrate at att $\lambda$ in host chromosomes (Howe, unpublished data). $\lambda c I 857 S 7$ helper phage and phages $\lambda c I$, $\phi 80 \mathrm{imm} \lambda c \mathrm{I}$, and $\phi 80$ were from the collections of $\mathrm{M}$. Howe and J. Adler. $\lambda c \mathrm{I} 857 \mathrm{psu}^{+} 2$ was obtained from H. Inokuchi.

Construction of LFT donors. Strain HK1100 was infected with $\lambda$ pMu526 or $\lambda$ pMu624 at a multiplicity of infection (MOI) of 5 , and $\lambda$-immune clones were selected by their ability to survive infection by $\lambda c I$ and $\phi 80 \mathrm{imm} \lambda c \mathrm{I}(20)$. Either a mixture of such $\lambda$-immune clones or purified clones were the source of low-frequency-transducing (LFT) lysates.

Isolation of transducing phages. LFT lysates were made by heat induction of the $\lambda c 185757 \mathrm{pMu}-\mathrm{Mu}$ dilysogens and lysis of the cells by chloroform. $\mathrm{Mu}$ resistant derivatives of recipient strains $\mathrm{M} 590$ (cheB $B_{0}$ ) $(3,34)$ and M524 $(\operatorname{motB})(2)$ were infected with a mixture of LFT lysate (MOI, 0.1) and $\lambda c I 857 S 7$ helper lysate (MOI, 10), and $\mathrm{Che}^{+}$or $\mathrm{Mot}^{+}$transductants were isolated by brushing the infected cells onto soft agar plates (2) and picking cells from distinct swarms, each of which represented a transductant clone. $\mathrm{Che}^{+}$ and $\mathrm{Mot}^{+}$transductants were heat induced, and the resulting lysates were tested (i) for the presence of plaque-forming phages able to grow on strain QD5003 (15), which is permissive for $S 7$ mutants (12), and (ii) for high-frequency transduction (HFT) of che $B_{0}{ }^{+}$or $\mathrm{mot}^{+}$by infection of $\lambda$-lysogenic derivatives of M590 or M524 at an MOI of 0.01 to 0.1. When such HFT activity was detected, the transducing phage was assayed for the presence of other bacterial genes by infecting the lambda lysogens of appropriate tester strains defective in other genes of the che cluster (strains W3623HflaIam88, M526, M524, R487cheA104, R487cheW113, AW569, R487cheX203,

TABLE 1. Bacterial strains

\begin{tabular}{|c|c|c|}
\hline Strain & Genotype & $\begin{array}{l}\text { Source and/or refer- } \\
\text { ence }\end{array}$ \\
\hline AW405 & lac gal ara leu thr his tonA tsx strA & 2 \\
\hline AW518 & lac gal ara leu thr his tonA tsx strA tsr & 30 \\
\hline AW569 & lac gal ara leu his tonA tsx strA tsr tar & $21,36,46$ \\
\hline HK1100 & $\operatorname{argE}$ strA flaG1540::Mu & This work \\
\hline M524 & lac gal ara leu thr his tonA tsx strA motB & 2 \\
\hline M526 & lac gal ara leu thr his tonA tsx strA motA & 2 \\
\hline M590 & lac gal ara leu thr his tonA tsx strA che $B_{0}$ & 3,34 \\
\hline MS1540 & recA argE his strA flaG1540::Mu & 42 \\
\hline PK25 & Hfr lac leu thr tonA azi & 18 \\
\hline R487cheA104 & lac gals ara leu thr his metF tonA strA rif che $A 104$ & 34; J. S. Parkinson \\
\hline R487cheB294 & lac gald ara leu thr his metF tonA strA rif cheB294 & 34; J. S. Parkinson \\
\hline R487che W113 & lac gals ara leu thr his metF ton $A$ str $A$ rif che $W 113$ & 34; J. S. Parkinson \\
\hline R487cheX203 & lac gals ara leu thr his metF tonA strA rif cheX 203 & 34; J. S. Parkinson \\
\hline R487che Y220 & lac gals ara leu thr his metF tonA strA rif che $Y 220$ & 34; J. S. Parkinson \\
\hline R487cheZ292 & lac gals ara leu thr his metF tonA strA rif cheZ292 & 34; J. S. Parkinson \\
\hline S159 & gal uvr & $35 ;$ M. Nomura \\
\hline S159 $(\lambda)$ & gal uvr $(\lambda)$ & This work \\
\hline $\mathrm{S} 159\left(\lambda c \mathrm{I} 857 \mathrm{psu}^{+} 2\right)$ & gal uvr $\left(\lambda c \mathrm{I} 857 \mathrm{psu}^{+} 2\right)$ & This work \\
\hline W3623H flaIam88 & gal trp his strA flaIam88 & This work \\
\hline
\end{tabular}


R487cheB294, R487cheY220, and R487cheZ292 to test flaI, motA, motB, cheA, cheW, tar, cheX, cheB, cheY, and $c h e Z$, respectively). The HFT lysates were also tested for the presence of plaque-forming transducing phages. Phages from 100 plaques from an HFT lysate were pooled and tested for $\mathrm{cheB}_{0}{ }^{+}$or $\operatorname{mot} \mathrm{B}^{+}$transducing activity. Since the titer of transducing activity in HFT lysates ranged from 10 to $200 \%$ of plaque titer, 100 plaques should have included plaques of transducing phage if the transducing phage was plaque forming. On the other hand, if the transducing phage was defective, only helper phage would have formed plaques, and the pooled phage would not have had transducing activity.

Titration of $\boldsymbol{c h e}^{+}$or mot $^{+}$transducing activity. HFT lysates were diluted by 10 -fold serial dilutions, and $10 \mu \mathrm{l}$ of each dilution was mixed with $50 \mu \mathrm{l}$ of a culture of $\lambda$-lysogenic recipient bacteria and brushed onto a soft agar plate (2). After incubation at $35^{\circ} \mathrm{C}$ overnight, the number of trails due to abortive transductants $(26,47)$ was counted.

Transfer of mutations onto גche4. Mutations from bacterial chromosomes can be put onto a transducing phage by a cross done in the following two steps (32): (i) integration of the transducing phage into the bacterial chromosome by recombination in the region of bacterial DNA homology, and (ii) excision of the transducing phage upon induction of phage development. Since $\lambda$ che 4 is defective in phage morphogenesis (see below), the following procedure was used to accomplish the two steps. $\lambda$ che 4 was separated from helper phage by repeated $\mathrm{CsCl}$ isopycnic centrifugation. To transfer the amber mutation cheA104 onto $\lambda$ che4, $\lambda$ che4 lysogens of R487cheA104 (28) were selected from $\lambda$ che4-infected cells. The lysogens were induced at $42^{\circ} \mathrm{C}$ to promote $\lambda$ che 4 excision and development and at the same time were infected with $\lambda c I 857 S 7$ helper phage to provide phage morphogenesis functions. The resulting lysate contained the original $\lambda$ che4, recombinant phages with the cheA104 mutation ( $\lambda$ che4-cheA104), and helper phages. Strain M590 (che $\left.B_{0}\right)$ was infected with the lysate at a low MOI $(<0.1)$ and with additional helper phage at a high MOI (>3), and Che ${ }^{+}$transductants were obtained on T-swarm plates (2). The $\mathrm{Che}^{+}$transductants were tested for $\mathrm{CheA}^{+}$and $\mathrm{CheB}_{0}^{+}$-HFT donor activity. Among the $\mathrm{CheB}_{0}{ }^{+}$transductant strains tested, those which yielded lysates with a high level of $\mathrm{CheB}_{0}^{+}$ transducing activity but with 1,000 - to 10,000 -fold less $\mathrm{CheA}^{+}$transducing activity were retained as double lysogens [for example, M590 ( $\lambda c 1857 S 7, \lambda c h e 4-$ cheA104)]. $\lambda$ che 4 phage strains carrying the amber mutations tar633 (36) and flaIam88 (isolated from W3623H after chi phage selection [22]) were constructed similarly.

Detection of phage-coded proteins in UV-irradiated cells. The $\lambda$ che 4 and helper phage $\lambda c I 857 S 7$ in a lysate were separated by $\mathrm{CsCl}$ isopycnic centrifugation (20) and dialyzed against $1 \mathrm{mM} \mathrm{MgSO}_{4}$ in 10 mM Tris-hydrochloride (pH 7.2). Bacterial strains $S 159, S 159(\lambda)$, and $S 159\left(\lambda c I 857 \mathrm{psu}^{+} 2\right)$ were cultured at $30^{\circ} \mathrm{C}$ in growth medium containing half-strength H1 salts (34) supplemented with $1 \mu \mathrm{g}$ of thiamine per $\mathrm{ml}, 0.2 \%$ glycerol, and $0.2 \%$ maltose to an optical density at $590 \mathrm{~nm}$ of 0.5 (35). The cells were washed by centrifugation, suspended in one-fifth the original volume of half-strength $\mathrm{H} 1$ salts solution, and irradiated with UV light under a germicidal lamp at a dose which lowered the incorporation of ${ }^{14} \mathrm{C}$-amino acids into protein to 1 to $3 \%$ of the incorporation observed in unirradiated cells. The UV-irradiated cells were mixed with the phage suspension to give an MOI of 5 and kept on ice for 5 to $10 \mathrm{~min}$. Then, $0.5 \mathrm{ml}$ of the mixture was diluted into $4 \mathrm{ml}$ of prewarmed growth medium and incubated at $30^{\circ} \mathrm{C}$ for $15 \mathrm{~min}$ with gentle shaking; $2 \mu \mathrm{Ci}$ of a uniformly ${ }^{14} \mathrm{C}$-labeled L-amino acid mixture (New England Nuclear Corp.) was added to the culture, which was incubated for $30 \mathrm{~min}$. The labeling was terminated by adding $0.5 \%$ Casamino Acids (Difco Laboratories), followed 5 min later by putting the culture on ice. The cells were pelleted by centrifugation, suspended in the sample buffer for electrophoresis (24), and incubated at $0^{\circ} \mathrm{C}$ for $15 \mathrm{~min}$. The solubilized proteins were electrophoresed in a polyacrylamide slab gel containing sodium dodecyl sulfate (24). After electrophoresis, the gel slab was processed for fluorography (25).

Labeling MCPs. Bacterial strains AW405 and AW518 (tsr) were grown in tryptone broth (2) at $30^{\circ} \mathrm{C}$ to an optical density at $590 \mathrm{~nm}$ of 0.45 , washed three times by centrifugation in a medium containing 10 $\mathrm{mM}$ potassium phosphate $(\mathrm{pH} 7.0)$ and $0.1 \mathrm{mM}$ EDTA, and suspended at a cell concentration to give an optical density at $590 \mathrm{~nm}$ of 2 in the same medium supplemented with $200 \mu \mathrm{g}$ of chloramphenicol per $\mathrm{ml}$ and $10 \mathrm{mM}$ DL-lactate (23). After incubation at $30^{\circ} \mathrm{C}$ for $5 \mathrm{~min}, \mathrm{~L}-\left[\mathrm{meth}_{\mathrm{H}}-^{3} \mathrm{H}\right]$ methionine (New England Nuclear Corp.) was added to the cell suspension to 4 $\mu \mathrm{M}$. After $30 \mathrm{~min}$ of incubation, the cells were pelleted by centrifugation and processed as described above for ${ }^{14} \mathrm{C}$-amino acid-labeled cells.

\section{RESULTS}

Rationale for transducing phage isolation. To isolate $\lambda$ specialized transducing phages carrying a particular gene, it is first necessary to obtain lysogens in which the $\lambda$ prophage is integrated close to the gene of interest. In this work we describe a general procedure which takes advantage of the random integration properties of bacteriophage $\mathrm{Mu}$ to isolate such lysogens. First, strains which contain a Mu prophage near the gene of interest are isolated. Then these $\mathrm{Mu}$ lysogenic strains are infected separately with $\lambda$ pMu phages lacking the $\lambda$ attachment site and the integrase gene but carrying a small amount of Mu DNA from the immunity end or the $\beta$ end of a Mu prophage inserted into lac DNA and located to the right of the $\lambda J$ gene. $\lambda \mathrm{pMu}$ lysogens arise due to integration of the infecting phage by homologous recombination between Mu DNA sequences present in the $\lambda$ phage and chromosome or, more rarely, between lac DNA sequences in the $\lambda$ phage and chromosome. Recombination between Mu DNA sequences results in the integration of the $\lambda$ pMu at one end of the Mu prophage (Fig. 1), resulting in a struc- 


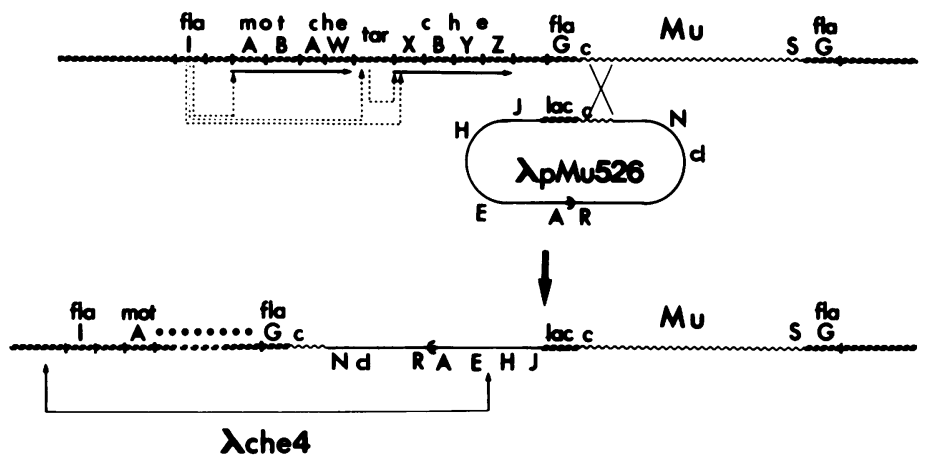

FIG. 1. Mechanism of generation of $\lambda$ che 4 transducing phage. This model involves the following two steps: (i) integration of $\lambda$ pMu526 into the chromosome of HK1100 by homologous recombination with the Mu prophage and (ii) excision of $\lambda$ che 4 by illegitimate recombination at the sites indicated by arrows. Straight lines represent $\lambda$ DNA, thin wavy lines represent $M u D N A$, and thick wavy lines represent host DNA. Regulatory circuits of che gene expression are shown at the upper left. Solid arrows indicate transcriptional units. mot $A$, motB, che $A$, and cheW are cotranscribed as the mocha operon $(34,43,44)$, and che $X$, che $B$, che $Y$, and cheZ are cotranscribed as the cheX operon $(34,44)$. Polar mutants of the cheX operon defective in the expression of the entire operon are called che $B_{0}$ mutants (34). The dashed arrows indicate positive regulation, either direct or indirect, of the genes. flaI gene activity is required for the expression of the mocha operon (43), tar gene (41), and cheX operon (41); tar gene activity is required for the expression of the cheX operon (Kondoh, unpublished data).

ture in which the $\lambda \mathrm{pMu}$ is flanked on one side by host sequences originally located at one end of the $\mathrm{Mu}$ prophage and on the other side by an intact $\mathrm{Mu}$ prophage. Integration of the $\lambda \mathrm{pMu}$ which carries the immunity end of Mu occurs at the immunity end of the resident $\mathrm{Mu}$ prophage, whereas integration of $\lambda \mathrm{pMu}$ carrying the $\beta$ end of $\mathrm{Mu}$ occurs at the other end of the Mu prophage. Because a given integrated $\lambda \mathrm{pMu}$ is flanked on only one side by host genes, it is able to make specialized transducing phages for only those host genes (i.e., those genes which were on only one side of the original $\mathrm{Mu}$ prophage). Lysogens containing the other $\lambda \mathrm{pMu}$ phage are able to make specialized transducing phages for host genes located at the other end of the original Mu prophage. This asymmetry of transducing phage isolation can be used to determine the orientation of the $\mathrm{Mu}$ prophage. Once such $\lambda \mathrm{pMu}$ lysogens are isolated, induction of the lysogens and isolation of transducing phages are performed in the usual manner.

Isolation of che transducing phages. Strain HK1100 with a Mu insertion in flaG was infected with the $\lambda \mathrm{pMu}$ transducing phage $\lambda \mathrm{pMu} 526$ or $\lambda \mathrm{pMu} 624$, and $\lambda \mathrm{pMu}$ lysogens were selected by their immunity to $\lambda$. $\lambda$ pMu 526 carries the immunity $(c)$ end of Mu prophage, whereas $\lambda$ pMu624 carries the $\beta$ end of Mu. In the lambdaimmune lysogens, $\lambda$ pMu phages should be integrated either at the site of the Mu prophage or in the lac region.

A mixture of lambda-immune cells representing more than 1,000 clones from each $\lambda \mathrm{pMu}$ infection was heat induced to promote $\lambda \mathrm{pMu}$ phage growth, and the resulting lysates were tested for $\mathrm{motB}^{+}$and $c h e B_{0}{ }^{+}$transducing activities. Transduction was assayed by determining the frequency of cells able to form trails (linear groups of microcolonies) on plates with very soft agar. Such trails result from the unilinear inheritance of $\mathrm{mot}^{+}$or $\mathrm{che}^{+}$transducing DNAs in motile abortive transductants arising from the nonmotile recipient strain $(26,47)$. The lysates from $\lambda$ pMu526 lysogens exhibited $\mathrm{mot}^{+}$and che ${ }^{+}$transduction activities at a frequency of approximately $10^{-5}$ per induced cell, whereas the lysates from $\lambda$ pMu624 lysogens showed no $\mathrm{mot}^{+}$ or $c h e^{+}$transducing activity. This observation indicated that the orientation of the $\mathrm{Mu}$ prophage insertion in flaG is the orientation shown in Fig. 1, where the immunity end is proximal to the mot and che genes ("+" orientation [17]).

A total of 20 independent $\lambda$ pMu526 lysogens of HK1100 each produced LFT lysates containing $\mathrm{mot}^{+}$and $\mathrm{cheB}_{0}{ }^{+}$transduction activities. HFT lysates were derived from $c h e B_{0}{ }^{+}$transductants which arose from infection with each LFT lysate, and each was found to be active in transducing all 10 genes ( flaI through cheZ [Fig. 1]) simultaneously. No HFT lysate contained plaque-forming transducing phages. The reason that all independent transducing phages selected for the proximal $c h e B_{0}{ }^{+}$marker contained the entire che cluster is not known.

One of the $c h e^{+}$transducing phages, designated $\lambda$ che4, was characterized in detail. This phage carries the following genes: flaI, motA, 
mot $B$, che $A$, che $W$, tar, cheX, che $B$, che $Y$, and cheZ. Figure 2 shows the distribution of plaqueforming and transducing phages resulting from $\mathrm{CsCl}$ isopycnic centrifugation of an induced lysate containing $\lambda$ che4 and helper phage $\lambda c I 857 S 7$. $\lambda$ che4 was titrated by the number of trails produced by MotB $^{+}$abortive transductants. MotB ${ }^{+}$transducing activity was maximum at a density of $1.489 \mathrm{~g} / \mathrm{cm}^{3}$, which coincided with a peak of absorbance, whereas the number of plaque-forming phage was maximum at a different density, namely, $1.508 \mathrm{~g} / \mathrm{cm}^{3}$. The observation that no peak of active phage accompanied the peak of transduction indicates that $\lambda$ che 4 is a defective phage. For comparable numbers of phage particles, as determined by the absorbance assay, similar amounts of plaque formation and trail formation were detected for helper

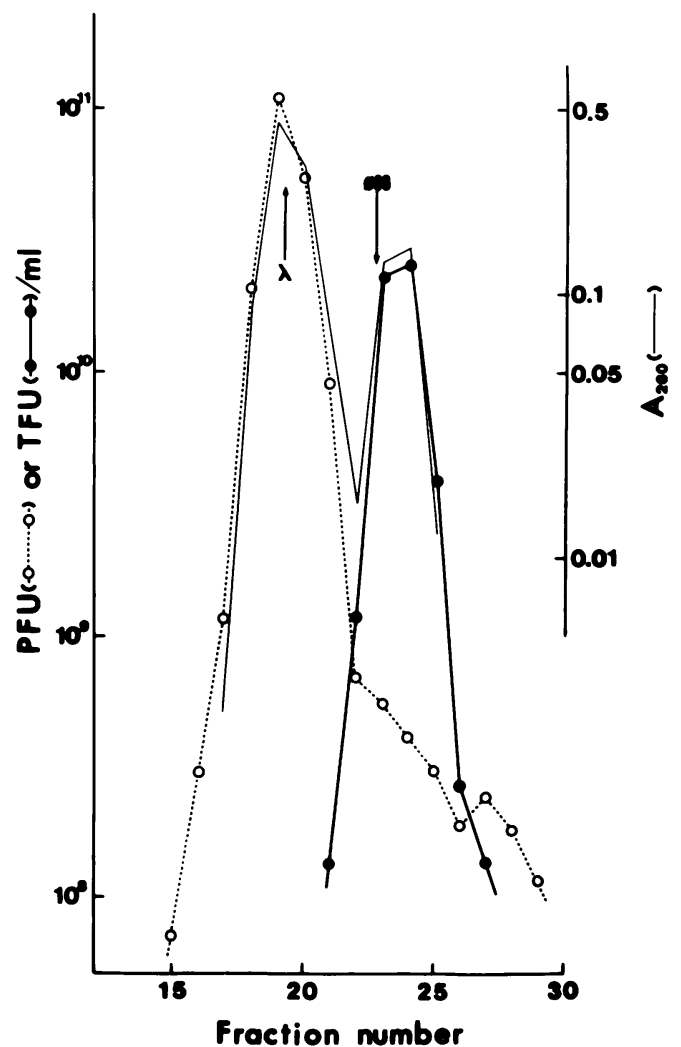

Fig. 2. $\mathrm{CsCl}$ isopycnic centrifugation of an HFT lysate of $\lambda$ che4. The centrifugation was carried out as described previously (20). Fractions were taken from the bottom of the tube, and absorbance at 260 $\mathrm{nm}\left(\mathrm{A}_{260}\right)$ and phage titer were measured. The titer of the active phage is expressed as plaque-forming units on strain QD5003, and the titer of the transducing phage is expressed as trail-forming units (TFU) on strain M524 $\lambda$ ). The positions of the density markers $\lambda\left(\rho=1.508 \mathrm{~g} / \mathrm{cm}^{3}\right)$ and $\phi 80\left(\rho=1.493 \mathrm{~g} / \mathrm{cm}^{3}\right)$ are also indicated. phages and $\lambda$ che 4 phages, respectively. This indicates that trail formation is an efficient and reliable assay for the presence of these defective transducing phages. From the particle density of $\lambda$ che4, the length of $\lambda$ che4 DNA is estimated to be about $83 \%$ of wild-type lambda DNA length (about 41 kilobase pairs). The effects of heat induction on single lysogens of $\lambda$ che 4 were also observed. Cells induced by incubation at $42^{\circ} \mathrm{C}$ elongated and, after chloroform was added, lysed without liberation of active phages. This observation suggests that endolysin production proceeds normally on the $\lambda$ che 4 genome and that the $\lambda$ DNA including genes $N$ through $R$ is preserved in $\lambda$ che4. Presumably, the defect of $\lambda$ che4 lies in morphogenetic genes which would be lost during the generation of $\lambda$ che 4 as shown in Fig. 1.

Proteins coded by the $\lambda$ che 4 transducing fragment. Proteins coded by $\lambda$ che 4 and $\lambda$ were labeled after infection of UV-irradiated cells and were analyzed by electrophoresis in polyacrylamide gels (Fig. 3 and 4). In the absence of $\lambda$ repressor in UV-irradiated cells, infection by $\lambda$ che 4 or $\lambda$ resulted in the synthesis of a number of phage-specific proteins (Fig. 3, lanes A and B, and Fig. 4, lanes $B$ and $C$ ). In $\lambda$ che4-infected cells the product of the $\lambda$ head gene $E$ was detected (Fig. 3, lane A), whereas the products of the tail genes $J$ and $H$ were not observed (Fig. 4 , lane B). This was the result expected if $\lambda$ che4 was generated as shown in Fig. 1 by replacing the $\lambda$ tail genes with transducing DNA.

Proteins 1 through 8 in Fig. 3 appeared only in $\lambda$ che4-infected cells and, therefore, were candidates for che-specific proteins. When UV-irradiated $\lambda$ lysogens were used as hosts, the presence of $\lambda$ repressor reduced the synthesis of $\lambda$ specific proteins (Fig. 3, lanes E and F) but did not affect the synthesis of proteins 1 through 6 and 8 (Fig. 3, lane F), thereby lending further support to the possibility that these are che proteins.

From a comparison of the molecular weights of the $\lambda$ che4-specific proteins with the molecular weights of proteins obtained from earlier studies of deleted $\lambda$ transducing phages $(41,44)$, it was possible to make tentative identifications of proteins $1,2,3,5,6$, and 8 as the products of specific che genes on the transducing fragment. Both proteins 1 and 2 (molecular weights, 76,000 and 66,000 ) were assigned to cheA, protein 3 (molecular weight, 62,000 ) was assigned to tar, protein 5 (molecular weight, 39,000) was assigned to mot $B$ and/or cheB, protein 6 (molecular weight, $29,000)$ was assigned to cheX, and protein 8 (molecular weight, 10,000 to 15,000 ) was assigned to cheW. Protein 7 (molecular weight, $24,000)$ may be the che $Z$ product; however, its 


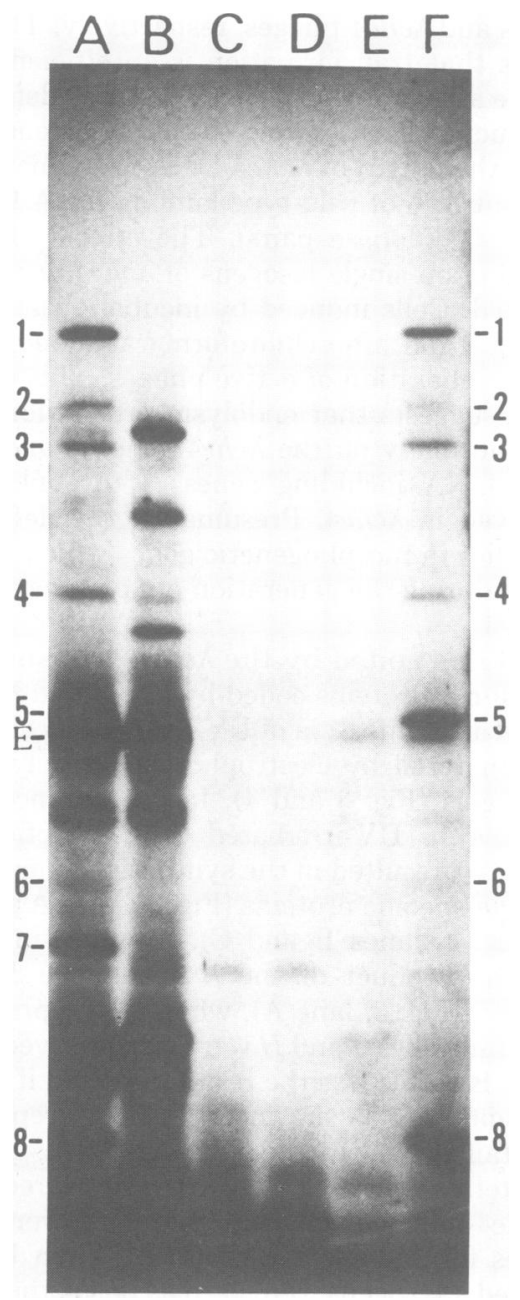

Fig. 3. Synthesis of proteins in UV-irradiated cells after infection with $\lambda$ che 4 or $\lambda$ cI857S7. Proteins were labeled, electrophoresed in a $10 \%$ polyacrylamide gel, and fluorographed. Lanes $A$ through $C$ show the results with strain $S 159$ (i.e., in the absence of lambda repressor), whereas lanes $D$ through $F$ show the results with strain $S 159$ ( $\lambda$ ) (i.e., in the presence of lambda repressor). Lanes $A$ and $F$, cells infected with $\lambda$ che4; lanes $B$ and $E$, cells infected with $\lambda$ cI857S7; lanes $C$ and $D$, cells not infected. Thus, a comparison of lanes $A$ and $F$ indicates the effect of lambda repressor upon $\lambda$ che4-directed protein synthesis, and a comparison of lanes $B$ and $E$ indicates the effect on $\lambda c I 85757$-directed protein synthesis. The positions of $\lambda$ che4-specific proteins 1 through 8 and lambda protein $E$ are indicated on the sides. To preserve detail in the low-molecular-weight region, the fluorogram was not exposed long enough to show lambda proteins $J$ and $H$ (see Fig. 4).

dependence on $\lambda$ gene expression leaves open the possibility that it is a $\lambda$ gene product instead. The gene for protein 4 (molecular weight, 48,000) was not identified. It is unlikely that either pro- tein 4 or protein 7 is the flaI gene product because introduction of an amber mutation of flaI into $\lambda$ che4 did not affect the synthesis of these proteins (data not shown).

Among the $\lambda$ che4-specific proteins, the highmolecular-weight proteins 1 through 3 were studied in detail. Separation of these proteins was increased by using $7 \%$ gels for electrophoresis. The migration rates of proteins 1 through 3 were compared with those of the MCPs (Fig. 4). Protein 1 migrated in a higher-molecular-

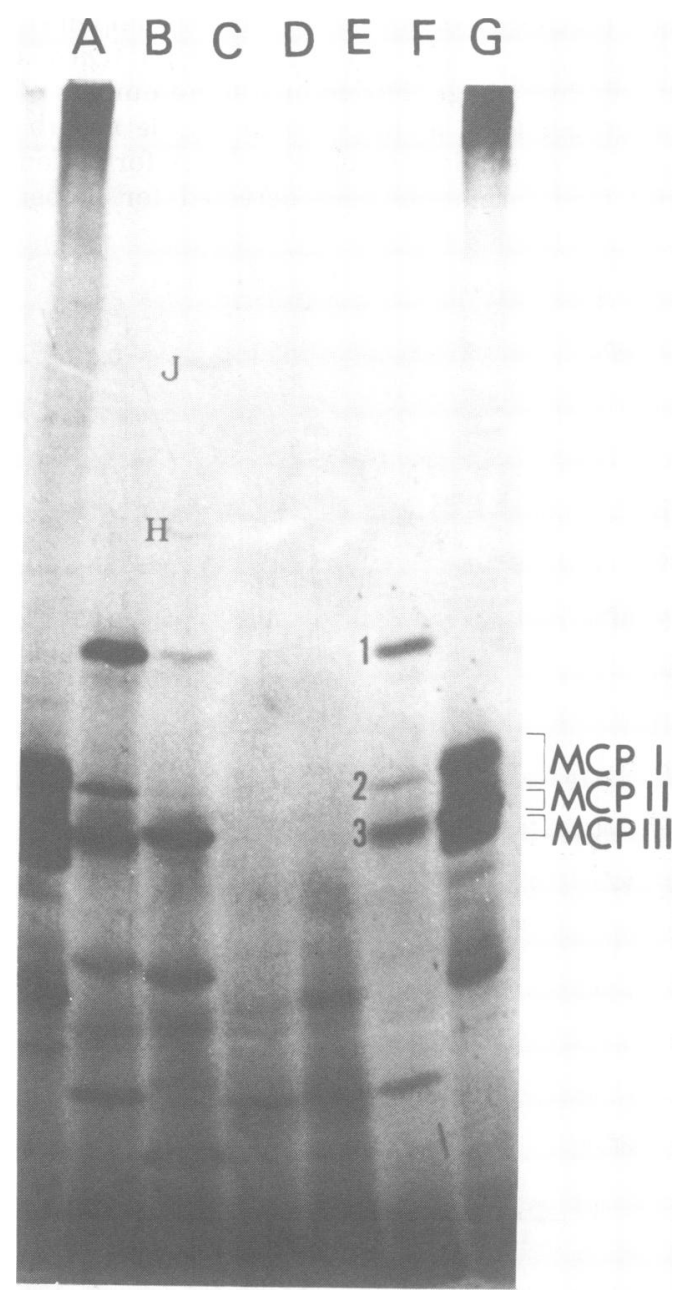

Fig. 4. Comparison of proteins 1 through 3 with MCPs. Electrophoresis was performed in a $7 \%$ poly. acrylamide gel. Lanes $A$ and $G$ contained methylated proteins of $A W 405$, and lanes $B$ through $F$ contained proteins synthesized and labeled in $U V$-irradiated bacteria. Lane B, S159 infected with $\lambda$ che4; lane $C$, $S 159$ infected with $\lambda$ cI857S7; lane $D$, uninfected $S 159$ $\lambda$ ); lane E, S159 ( $\lambda$ ) infected with $\lambda$ cI857S7; lane F, $S 159(\lambda)$ infected with $\lambda$ che4. The positions of proteins 1 through 3, MCPI through MCPIII, and lambda proteins $J$ and $H$ are marked. 
weight region than the MCPs, whereas protein 2 was located in the MCPI region of the gel. Deletion analysis has suggested that proteins 1 and 2 are both coded by the single cheA gene (44). This hypothesis was tested by examining the effect of a cheA amber mutation on $\lambda$ che4directed protein synthesis (Fig. 5). The amber mutation cheA104 eliminated synthesis of both proteins 1 and 2 and created a new protein, protein " $a$," which is probably the amber fragment (molecular weight, 67,000). The simplest interpretation of these data is that the cheA gene codes for both proteins 1 and 2 . It should be noted that the presumptive amber fragment

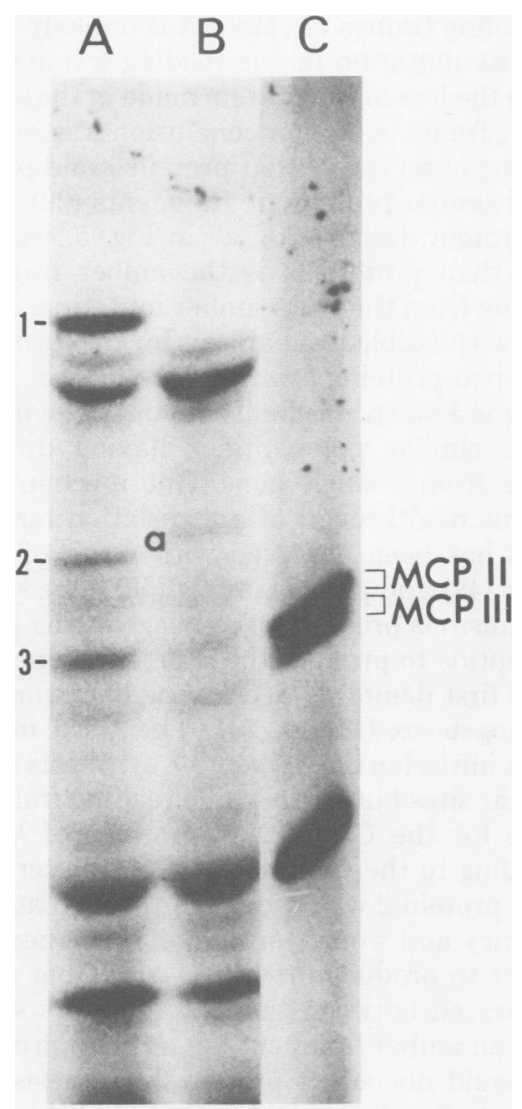

Fig. 5. Effect of the amber mutation cheA104 on proteins 1 and 2. UV-irradiated strain S159 $(\lambda)$ was

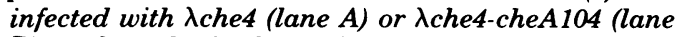
$B)$, and synthesized proteins were labeled and electrophoresed in a $7 \%$ polyacrylamide gel. Synthesis of proteins 1 and 2 was abolished by the cheA104 $\mathrm{mu}$ tation, but a new protein (labeled a) was observed. There was an unusually high background of host protein synthesis in this particular experiment. Lane $C$ contained methylated proteins of strain AW518, which lacks MCPI. Protein 3 is located at the same place as MCPII. "a" has a higher molecular weight than protein 2. This point is discussed below.

Protein 3 migrated to the same place as MCPII but not MCPI or MCPIII in 7\% gels (Fig. 4 and 5). Since it has been suggested that tar is the structural gene for MCPII $(41,44-46)$, we studied the effect of a tar amber mutation on $\lambda c h e 4$-directed protein synthesis (Fig. 6). In $s u-$ cells, the tar 633 amber mutation eliminated synthesis of protein 3 . The synthesis of this protein was recovered in cells containing the amber suppressor $\mathrm{su}^{+} 2$ (supE). This was the result expected if tar is the structural gene for protein 3 (MCPII).

\section{DISCUSSION}

$\lambda c h e 4$, a $\lambda$ specialized transducing phage carrying the 10 known genes of the che cluster (41.5 to $42.5 \mathrm{~min}$ ), was isolated by using $\mathrm{Mu}$ DNA homology to direct the integration of $\lambda$ near che. Proteins synthesized by $\lambda$ che4 in UV-irradiated cells were separated by polyacrylamide gel electrophoresis, and che4-specific proteins were tentatively identified. Similar analyses of the proteins synthesized by $\lambda$ che4 phages carrying amber mutations in the cheA or tar genes allowed the identification of the cheA gene products as proteins 1 (molecular weight, 76,000 ) and 2 (molecular weight, 66,000) and the tar gene product

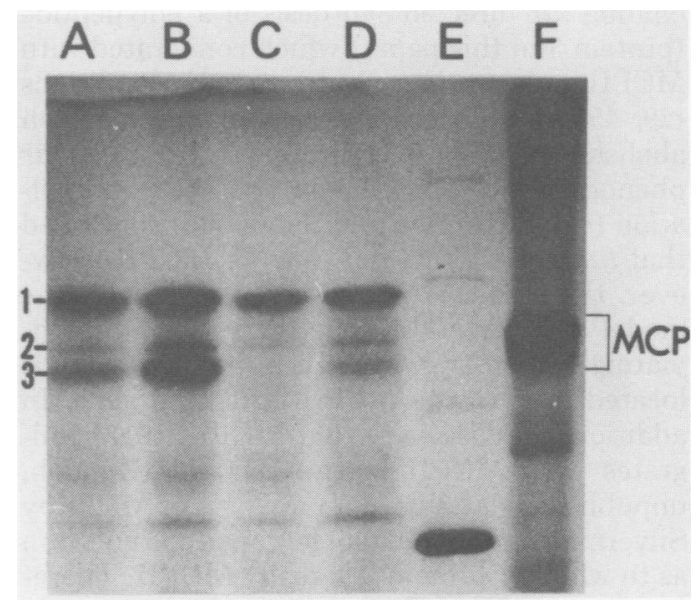

Fig. 6. Effect of the amber mutation tar633 on protein 3 and suppression by $\mathrm{su}^{+} 2$ amber suppressor. $U V$-irradiated cells were infected with transducing phages, and synthesized proteins were labeled and electrophoresed in a $12 \%$ polyacrylamide gel. Lane A, $S 159(\lambda)$ infected with $\lambda$ che4; lane $B, S 159\left(\lambda p s u^{+} 2\right)$ infected with $\lambda$ che4; lane $C, S 159(\lambda)$ infected with $\lambda$ che4-tar633; lane $\left.D, S 159 \lambda_{p s u^{+}} 2\right)$ infected with $\lambda$ che4-tar633; lane $E$, proteins isolated from lambda phage particles; lane $F$, methylated proteins of strain $A W 405$. The positions of proteins 1 through 3 and the MCPs are indicated at the sides of the gel. 
as protein 3 (molecular weight, 62,000 ), which is MCPII.

Previous analyses of the polypeptides coded by genes involved in bacterial chemotaxis were performed by Simon and co-workers, using deleted derivatives of $\lambda$ specialized transducing phages carrying portions of the che region of the $E$. coli chromosome $(41,44,45)$. In these studies individual proteins were assigned to specific genes by correlating the presence of the protein with the presence of complementing activity for specific host mutations. Using such evidence, these investigators concluded that the che $A$ gene codes for two proteins corresponding to proteins 1 and 2 of this study $(41,44)$ and that the $t a r$ gene codes for a protein corresponding to protein 3 (45). Although such correlations significantly decrease the number of candidate proteins which may be coded by specific genes, they do not prove conclusively that the observed proteins are those specific gene products. For example, the deletions might remove not only the known gene but also an adjacent, as-yet-unidentified gene which actually codes for the observed protein.

Previous analyses of tar mutants showed that they are devoid of taxis toward or away from a class of chemicals $(36,45,46)$ and that they are also defective in methylation of MCPII $(45,46)$. Since transducing phages carrying a region including tar directed synthesis of a polypeptide (protein 3 in this paper) which comigrated with MCPII under various electrophoretic conditions $(29,45)$ and since a deletion mutation which abolished synthesis of protein 3 led to a tar phenotype in both behavior and MCP methylation (44), Silverman and Simon (45) concluded that tar is the structural gene for MCPII. However, the evidence is also compatible with the model that tar is the gene for a specific methylating system for MCPII (protein 3) and is located next to the MCPII structural gene. In addition, the discovery that MCPIII (21) comigrates with MCPII in the $12 \%$ gel (Kondoh, unpublished data) used for analysis of MCPs by Silverman and Simon (45) left open the question as to whether protein 3 is really MCPII. Therefore, we analyzed the effect of a tar nonsense mutation on these proteins by using gels which effectively separated the MCPs. The tar nonsense mutation eliminated synthesis of protein 3 (Fig. 6), which comigrated with MCPII but not with MCPIII (Fig. 4 and 5). These results support the conclusion that tar codes for MCPII; however, it should be noted that because of possible polarity of the amber mutation, they also do not conclusively rule out the unlikely possibility that an adjacent unidentified gene may be involved in protein 3 production.
The previous assignment of proteins 1 and 2 to the cheA gene was based on analyses of deleted derivatives of $\lambda$ transducing phages (41, 44). Because the amount of DNA corresponding to che $A$ was too small to code for two contiguous proteins of the sizes of proteins 1 and 2, it was suggested that the proteins were either coded by overlapping genes or were related to one another by processing of a single polypeptide chain (44). The observation that a nonsense mutation in che $A$ abolishes the syntheses of both protein 1 (molecular weight, 76,000) and protein 2 (molecular weight, 66,000) (Fig. 5) confirms the assignment of these proteins to the che $A$ gene. It also argues against the hypothesis that the proteins are made from overlapping genes read in different reading frames (4), since it is unlikely that a nonsense mutation in one reading frame would lead to the loss of the protein made in the second reading frame. A similar conclusion was reached from the observation that protein 1 and protein 2 yield similar proteolytic fragments (29). If the new protein designated " $a$ " in Fig. 5, which is larger than protein 2, is the amber fragment resulting from the che $A$ amber mutation, it suggests two possible mechanisms for the formation of the two proteins from the cheA gene. There are three known mechanisms for the synthesis of two similar polypeptides having different lengths from a single gene. One mechanism is frequent readthrough of a translation terminator, as has been observed with genes of RNA phages (49) and phage lambda (50). The second mechanism is proteolytic cleavage of the longer polypeptide to produce the shorter polypeptide, as was first demonstrated during bacteriophage morphogenesis $(11,14,24)$. The third mechanism is initiation of polypeptide synthesis at two different sites but in the same reading frame, as is true for the $\mathrm{C}$ and Nu3 proteins of $\lambda$ (38). According to the first mechanism, the terminator for protein 2 would be read through at some frequency and would terminate at the next terminator to produce protein 1 . An amber mutation located between these terminators should create an amber fragment longer than protein 2 but should not affect protein 2 synthesis. The fact that the cheA104 mutation abolished synthesis of protein 2 makes the readthrough hypothesis less likely. Both the second and third mechanisms remain possible.

In this paper we describe a general method for constructing transducing phages for any given gene of $E$. coli by using $\lambda \mathrm{pMu}$ phages. The essence of the method is to integrate the $\lambda \mathrm{pMu}$ into or near the genes of interest by using homologous recombination with a Mu prophage. The use of a Mu prophage for the site of $\lambda \mathrm{pMu}$ integration was described for the first time by 
Casadaban (8). In this report we describe the isolation of transducing phages for chemotaxis genes by this method. The same approach has been used to isolate transducing phages for the ara genes of E. coli (B. R. Paul, L. Parada, and M. M. Howe, unpublished data) and for the nif genes of Klebsiella pneumoniae (27). Since the $\lambda \mathrm{pMu}$ phages used here also carry a part of the lac operon, they might integrate either in the Mu prophage or in the lac operon; however, we found that integration into the lac region was rare and did not interfere with the isolation of LFT donors. LFT donors were readily obtained by using a mixture of $\lambda \mathrm{pMu}$ lysogens or by purifying a moderate number of the lysogens.

A $\lambda$ pMu phage carrying the immunity $(c)$ end of $\mathrm{Mu}$ was effective in producing transducing phages for che genes, whereas a $\lambda$ pMu carrying the $\beta$ end was not. This observation indicated that the insertion of the Mu prophage in flaG in strain MS1540 was in the + orientation (17). This kind of analysis may be extended to other cases to assess the orientation of $\mathrm{Mu}$ prophage insertion if genes near the Mu prophage are known. Conversely, if the polarity of a Mu prophage insertion is known, for instance by the method of chromosome mobilization (51), the arrangement of bacterial genes in the vicinity of the $\mathrm{Mu}$ prophage can be determined by the spectrum of transducing phages derived from $\lambda \mathrm{pMu}$ phages carrying the $c$ or $\beta$ ends of $\mathrm{Mu}$.

The usefulness of $\lambda \mathrm{pMu}$ phages as vehicles of specialized transduction is based on the fact that Mu integrates at virtually any place on the chromosome irrespective of base sequences $(7,9)$. This situation is in sharp contrast to integration of $\lambda$ at secondary attachment sites, which are not randomly distributed (39), or to integration of $F$ or drug resistance elements, which may be limited to a smaller number of sites than $\mathrm{Mu}$ $(10,19)$. If a $\mathrm{Mu}$-induced mutation near the gene of interest is not available, $\mathrm{Mu}$ prophage insertion within the desired region can be achieved by localized mutagenesis by $\mathrm{Mu}(48)$. Theoretically, it may also be possible to derive transducing phages starting from a random mixture of $\mathrm{Mu}$ lysogens. By further lysogenizing such $\mathrm{Mu}$ lysogens with $\lambda \mathrm{pMu}$ phages, a cell population would be obtained in which $\lambda \mathrm{pMu}$ phages are distributed rather uniformly over the entire $E$. coli chromosome. An induced lysate of such a population should contain a mixture of transducing phages carrying various portions of the $E$. coli genome. Such an approach has been successful for isolating transducing phages from secondary attachment sites for lambda (37). This method for isolating specialized transducing phages by taking advantage of the random- ness of $\mathrm{Mu}$ integration should be widely applicable in in vivo genetic engineering.

\section{ACKNOWLEDGMENTS}

We thank S. J. Kleene for conducting some of the experiments with UV-irradiated cells and J. Adler for support and encouragement.

This work was supported by the College of Agricultural and Life Sciences, University of Wisconsin-Madison, by Public Health Service grant AI08746 from the National Institutes of Health and National Science Foundation grant PCM75-21007 to J. Adler, and by Public Health Service grant AI12731 from the National Institutes of Health and National Science Foundation grant PCM75-02465 to M.M.H. H.K. was a Postdoctoral Fellow in Cancer Research supported by grant DRG110-F from the Damon Runyon-Walter Winchell Cancer Fund. B.R.P. was the recipient of National Science Foundation Undergraduate Research Participation Award SPI7683456. M.M.H. was the recipient of a Public Health Service Research Career Development Award from the National Institutes of Health.

\section{LITERATURE CITED}

1. Adler, J., and B. Templeton. 1963. The amount of galactose genetic material in $\lambda \mathrm{dg}$ bacteriophage with different densities. J. Mol. Biol. 7:710-720.

2. Armstrong, J. B., and J. Adler. 1967. Genetics of motility in Escherichia coli: complementation of paralyzed mutants. Genetics 56:363-373.

3. Armstrong, J. B., and J. Adler. 1969. Complementation of nonchemotactic mutants of Escherichia coli. Genetics 61:61-66.

4. Barrell, B. G., G. N. Air, and C. A. Hutchison III. 1976. Overlapping genes in bacteriophage $\phi \times 174$. Nature (London) 264:34-41.

5. Blattner, F. R., B. G. Williams, A. E. Blechl, K. Denniston-Thompson, H. E. Faber, L.-A. Furlong, D. J. Grunwald, D. O. Kiefer, D. D. Moore, J. W. Schumm, E. L. Sheldon, and O. Smithies. 1977. Charon phages: safer derivatives of bacteriophage $\lambda$ for DNA cloning. Science 196:161-169.

6. Bukhari, A. I., and B. Allet. 1975. Plaque-forming $\lambda$-Mu hybrids. Virology 63:30-39.

7. Bukhari, A. I., and D. Zipser. 1972. Random insertion of Mu-1 DNA within a single gene. Nature (London) New Biol. 236:240-243.

8. Casadaban, M. J. 1976. Transposition and fusion of the lac genes to selected promoters in Escherichia coli using bacteriophage lambda and Mu. J. Mol. Biol. 104: 541-555.

9. Daniell, E., R. Roberts, and J. Abelson. 1972. Mutations in the lactose operon caused by bacteriophage Mu. J. Mol. Biol. 69:1-8.

10. Davidson, N., R. C. Deonier, S. Hu, and E. Ohtsubo. 1974. Electron microscope heteroduplex studies of sequence organization of $F$ and F-primes and the sequences involved in Hfr formation, p. 56-65. In D. Schlessinger (ed.), Microbiology-1974. American Society for Microbiology, Washington, D. C.

11. Dickson, R. C., S. L. Barnes, and F. A. Eiserling. 1970. Structural proteins of bacteriophage T4. J. Mol. Biol. 53:461-473.

12. Goldberg, A. R., and M. Howe. 1969. New mutations in the $S$ cistron of bacteriophage lambda affecting host cell lysis. Virology 38:200-201.

13. Gottesman, S., and J. R. Beckwith. 1969. Directed transposition of the arabinose operon: a technique for the isolation of specialized transducing bacteriophages for any Escherichia coli gene. J. Mol. Biol. 44:117-127.

14. Hosoda, J., and R. Cone. 1970. Analysis of T4 phage proteins. I. Conversion of precursor proteins into lower 
molecular weight peptides during normal capsid formation. Proc. Natl. Acad. Sci. U.S.A. 66:1275-1281.

15. Howe, M. M. 1973. Prophage deletion mapping of bacteriophage Mu-1. Virology 54:93-101.

16. Howe, M. M. 1973. Transduction by bacteriophage $\mathrm{Mu}$. Virology 55:103-117.

17. Howe, M. M., and E. G. Bade. 1975. Molecular biology of bacteriophage Mu. Science 190:624-632.

18. Kahn, P. L. 1968. Isolation of high-frequency recombining strains from Escherichia coli containing the V colicinogenic factor. J. Bacteriol. 96:205-214.

19. Kleckner, N., J. Roth, and D. Botstein. 1977. Genetic engineering in vivo using translocatable drug-resistance elements. New methods in bacterial genetics. J. Mol. Biol. 116:125-159.

20. Kondoh, H. 1977. Isolation and characterization of nondefective transducing lambda bacteriophages carrying fla genes of Escherichia coli K-12. J. Bacteriol. 130: 736-745.

21. Kondoh, H., C. B. Ball, and J. Adler. 1979. Identification of a methyl-accepting chemotaxis protein for the ribose and galactose chemoreceptors of Escherichia coli. Proc. Natl. Acad. Sci. U.S.A. 76:260-264.

22. Kondoh, H., and H. Ozeki. 1976. Deletion and amber mutants of fla loci in Escherichia coli K-12. Genetics 84:403-421.

23. Kort, E. N., M. F. Goy, S. H. Larsen, and J. Adler. 1975. Methylation of a membrane protein involved in bacterial chemotaxis. Proc. Natl. Acad. Sci. U.S.A. 72: $3939-3943$.

24. Laemmli, U. K. 1970. Cleavage of structural proteins during the assembly of the head of bacteriophage T4. Nature (London) 227:680-685.

25. Laskey, R. A., and A. D. Mills. 1975. Quantitative film detection of ${ }^{3} \mathrm{H}$ and ${ }^{14} \mathrm{C}$ in polyacrylamide gels by fluorography. Eur. J. Biochem. 56:335-341.

26. Lederberg, J. 1956. Linear inheritance in transductional clones. Genetics 41:845-871.

27. MacNeil, D., M. M. Howe, and W. J. Brill. 1980. Isolation and characterization of $\lambda$ specialized transducing phages carrying the Klebsiella pneumoniae nif (nitrogen fixation) genes. J. Bacteriol. 141:1264-1271.

28. Magazin, M., M. Howe, and B. Allet. 1977. Partial correlation of the genetic and physical maps of bacteriophage Mu. Virology 77:677-688.

29. Matsumura, P., M. Silverman, and M. Simon. 1977. Synthesis of mot and che gene products of Escherichia coli programmed by hybrid ColE1 plasmids in minicells. J. Bacteriol. 132:996-1002.

30. Mesibov, R., and J. Adler. 1972. Chemotaxis toward amino acids in Escherichia coli. J. Bacteriol. 112:315326.

31. O'Day, K., D. Schultz, W. Ericsen, L. Rawluk, and M. Howe. 1979. Correction and refinement of the genetic map of bacteriophage Mu. Virology 93:320-328.

32. Oka, A., H. Ozeki, and J. Inselburg. 1971. Integration and excision of $\phi 80 \mathrm{pt}$ prophage in Escherichia coli. I. Replacement of tryptophan genes of $\phi 80$ pt with the host alleles through the lysogenic process. Virology 46: 556-566.

33. Parkinson, J. S. 1977. Behavioral genetics in bacteria.
Annu. Rev. Genet. 11:397-414.

34. Parkinson, J. S. 1978. Complementation analysis and deletion mapping of Escherichia coli mutants defective in chemotaxis. J. Bacteriol. 135:45-53.

35. Ptashne, M. 1967. Isolation of the $\lambda$ phage repressor. Proc. Natl. Acad. Sci. U.S.A. 57:306-313.

36. Reader, R. W., W.-W. Tso, M. S. Springer, M. F. Goy, and J. Adler. 1979. Pleiotropic chemotaxis mutants of Escherichia coli. J. Gen. Microbiol. 111:363-374.

37. Schrenk, W. J., and R. A. Weisberg. 1975. A simple method for making new transducing lines of coliphage $\lambda$. Mol. Gen. Genet. 137:101-107.

38. Shaw, J. E., and H. Murialdo. 1980. Morphogenetic genes $\mathrm{C}$ and Nu3 overlap in bacteriophage $\lambda$. Nature (London) 283:30-35.

39. Shimada, K., R. A. Weisberg, and M. E. Gottesman. 1972. Prophage lambda at unusual chromosomal sites. I. Location of the secondary attachment sites and the properties of lysogens. J. Mol. Biol. 63:483-503.

40. Signer, E. 1968. Lysogeny: the integration problem. NAnnu. Rev. Microbiol. 22:451-488.

41. Silverman, M., P. Matsumuta, M. Hilmen, and $M$. Simon. 1977. Characterization of lambda Escherichia coli hybrids carrying chemotaxis genes. J. Bacteriol. 130:877-887.

42. Silverman, M., and M. Simon. 1973. Genetic analysis of bacteriophage $\mathrm{Mu}$-induced flagellar mutants in Escherichia coli. J. Bacteriol. 116:114-122.

43. Silverman, M., and M. Simon. 1976. Operon controlling motility and chemotaxis in $E$. coli. Nature (London) 264:577-580.

44. Silverman, M., and M. Simon. 1977. Identification of polypeptides necessary for chemotaxis in Escherichia coli. J. Bacteriol. 130:1317-1325.

45. Silverman, M., and M. Simon. 1977. Chemotaxis in Escherichia coli: methylation of che gene products. Proc. Natl. Acad. Sci. U.S.A. 74:3317-3321.

46. Springer, M. S., M. F. Goy, and J. Adler. 1977. Sensory transduction in Escherichia coli: two complementary pathways of information processing that involve methylated proteins. Proc. Natl. Acad. Sci. U.S.A. 74: 3312-3316.

47. Stocker, B. A. D. 1956. Abortive transduction of motility in Salmonella: a non-replicated gene transmitted through many generations to a single descendant. J. Gen. Microbiol. 15:575-598.

48. Tabor, H. E., W. Hafner, and C. W. Tabor. 1977. Localized mutagenesis with bacteriophage $\mathrm{Mu}$ : method for increasing the frequency of specific bacterial mutants. J. Bacteriol. 132:359-361.

49. Weiner, A. M., and K. Weber. 1971. Natural readthrough at the UGA termination signal of $Q \beta$ coat protein cistron. Nature (London) New Biol. 234:206209.

50. Yates, J. L., W. R. Gette, M. E. Furth, and M. Nomura. 1977. Effects of ribosomal mutations on the readthrough of a chain termination signal: studies on the synthesis of bacteriophage $\lambda O$ gene protein in vitro. Proc. Natl. Acad. Sci. U.S.A. 74:689-693.

51. Zeldis, J. B., A. I. Bukhari, and D. Zipser. 1973. Orientation of prophage Mu. Virology 55:289-294. 